Iğdır Üniversitesi Fen Bilimleri Enstitüsü Dergisi, 11(3): 1887-1895, 2021

Elektrik Elektronik Mühendisliği / Electrical Electronic Engineering

ISSN: 2146-0574, eISSN: 2536-4618

Araştırma Makalesi / Research Article

Geliş tarihi / Received: 03.10.2020

DOI: $10.21597 /$ jist.804591

Kabul tarihi / Accepted: 10.04.2021

Atıf İçin: Karakulak E, Korkmaz Tan R, Mutlu R, 2021. Lotka-Volterra Denklemleri için STM32F429 Discovery Board Tabanlı Emülatör. Iğdır Üniversitesi FenBilimleri Enstitüsü Dergisi, 11(3): 1887-1895.

To Cite: Karakulak E, Korkmaz Tan R, Mutlu R, 2021. STM32F429 Discovery Board-Based Emulator for LotkaVolterra Equations. Iğdır Üniversitesi FenBilimleri Enstitüsü Dergisi, 11(3): 1887-1895.

\title{
STM32F429 Discovery Board-Based Emulator for Lotka-Volterra Equations
}

\section{Ertuğrul KARAKULAK ${ }^{1}$, Rabia KORKMAZ TAN²*, Reşat MUTLU ${ }^{3}$}

\begin{abstract}
Lotka-Volterra equations are commonly used in prey-predator population studies. Simulation programs are commonly used to produce solutions of Lotka-Volterra equations and to examine their initial value dependendence. In literature, chaotic waveform generators, ECG and EEG generators have been made and used for research and education. To the best of our knowledge, such an electrical circuit to produce the Lotka-Volterra waveforms does not exist. Such a circuit can be made using either analog or digital circuit components. However, such a device may be used for education in classroom and also to prove concepts by population researchers. In this study, implementation and experimental verification of the microcontroller-based circuit which solves LotkaVolterra equations in real time and produces its waveforms are presented. Euler method is used to solve the equation system in discrete time. Presented design has been implemented using an STM32F429 Discovery Board, two DACs and four opamps. The microcontroller sends the signals to the outputs of the circuit using digital-to-analog converters and opamps. The waveforms acquired experimentally from the implemented circuit outputs matches well with those obtained from numerical simulations.
\end{abstract}

Keywords: Lotka-Volterra equations, prey-predator simulations, system dynamics, microcontrollerbased design.

${ }^{1}$ Ertuğrul KARAKULAK (Orcid ID: 0000-0001-5937-2114), Namık Kemal University, Vocational School of Technical Sciences, Tekirdağ, Turkey

${ }^{2}$ Rabia KORKMAZ TAN (Orcid ID: 0000-0002-3777-2536), Namık Kemal University, Çorlu Engineering Faculty, Computer Engineering Department, Tekirdağ, Turkey

${ }^{3}$ Reşat MUTLU (Orcid ID: 0000-0003-0030-7136), Namık Kemal University, Çorlu Engineering Faculty, Electronics and Communication Engineering Department, Tekirdağ, Turkey

*Sorumlu Yazar/Corresponding Author: Rabia KORKMAZ TAN, e-mail: rkorkmaz@nku.edu.tr 


\section{INTRODUCTION}

A group of problems that have been largely studied in recent years within the scope of stability analysis in non-linear systems is the predator-prey system, which models the interaction of two or more species living in a common environment. Some of the species prey on the others and also the birth and death rates of species are expressed in parameters. The first model introduced in this respect is the Lotka-Volterra model (Lotka, 1926). This is a very important model as it is the starting point of more complex systems. But the model does not reflect the reality because of stability problems (Gleria et al. 2001). The model is important in terms of the disappearance of stability, the system shifting to chaotic behavior, and the analysis of limit cycles that exhibit structural stability and has been the subject of many studies.

An analysis of the Lotka-Volterra model gives out two populations. The predator species, one of the populations in this model, feed on the prey species in this mechanism. And the prey species feed on the food in the surroundings. The predator-prey model was first developed to analyze the changes in shark (predator) and fish (prey) populations (Volterra, 1978). Several calculations were made to find out population densities in this model. In the settings with no predator population, the change in prey population will increase at a ratio of $\mathrm{dx} / \mathrm{dt}=\mathrm{ax}$ for $\mathrm{a}>0$. In the settings with no prey population, the predator population will decrease at a ratio of $d y / d t=-d y$ for $d>0$. The ratios of increase and decrease will change according to the frequency with which the species meet and interact once they are in the same setting. This value is considered to be proportional to the value of x.y. In that case, while the prey ratio decreases at an additional rate of -bxy for $b>0$, the predator population increases at an additional rate of of cxy for $c>0$. When these values are combined as a single equation, the following equation system is obtained for finding out the prey and predator density (Scheiner, 1996):

$$
\begin{aligned}
\mathrm{dx} / \mathrm{dt} & =\mathrm{ax}-\mathrm{bxy} \\
\mathrm{dy} / \mathrm{dt} & =\mathrm{cxy}-\mathrm{dy}
\end{aligned}
$$

Because of their dependence upon the factors of time and location, mathematical models are established using ordinary differential equations, difference equations or partial differential equations. The dynamic systems defined by these equations are grouped into two as the discrete and the continuous systems, depending on time. While the natural events involving discrete systems are modeled using difference equations, continuous systems are modeled using differential equations. The above described Lotka-Volterra model is an example of continuous systems.

Modeled using differential equations, the predator-prey systems play an important part in analyzing the dynamics of biological populations in mutual interaction. Consequently, many researchers have analyzed the dynamic structure of the predator-prey models since their first introduction. Several different structures such as Hopf bifurcation, limit cycle, and chaotic behavior have been observed in these analyses.

The current studies have been examined as follows. Lotka and Volterra (1) have examined the population dynamics among species of organisms and set their models which were regarded as the ground for ecological processes. Further studies still continue to be conducted based on these models. Expressed by a system of differential equations, the Lotka-Volterra model was built on Liouville's theorem and a global essential for 'motion', as proved by Kerner (Kerner, 1957). A statistical mechanics of Lotka-Volterra model was advanced on this principle where the approach of microcanonical and canonical ensembles was proposed to Lotka-Volterra model along with a concept of temperature. Building on the studies of Kerner; Goel, Maitra, and Montroll reported a fine analysis related to the interacting communities (Goel et al., 1971). They strived to find out the outstanding 
properties of populations that are very indifferent to the first conditions and the details of the hypothesis data. Lotka-Volterra model was considered as a leading principle while doing a 'statistical mechanical' analysis of changes in the population. But the parameters specifying the dynamical system are expressed on a group basis, rather than on an individual basis in Lotka-Volterra type model. 'Stepping stone model' was established by Kimura (Kimura and Weiss, 1964). in the field of population genetics. This model was constituted to measure the decline of genetic correlation in line with distance in a population structured in a geographical way. Several studies have been conducted on the basis of the stepping stone model to examine the relationship between the geographical structure and genetic correlation in species. But no in-depth studies were conducted involving the individual characteristics and individuals' interactions. 'SIR model' (susceptible, infected, recovered) was launched by R.Kermack and McKendrick (Kermack and Mckendrick, 1927) in the field of dynamical epidemiology. 'SIR model' may be considered as a type of Lotka-Volterra model. Since the prey or the host causes an increase in the frequency of the predator or the infected, $\mathrm{S}$ and $\mathrm{R}$ may be correlated with two peculiar conditions of the prey or the host. In recent years, on the other hand, the growing interest in discrete population models which are expressed using the difference equations has been reflected in the studies involving predator-prey systems. While Boshan and Jiejie (Boshan and Jiejie, 2012) examined the dynamic structure of a ratio-dependent predator-prey model, discretized by Euler's method, using the Center Manifold Theorem for difference equations; Zhang and Boshan (Zhang et al., 2014) done research on the discrete-time predator-prey biological economical system. Elabbasy, Elsadany, and Zhang have proven the existence of a stable point of balance in a discrete and reduced Lorenz system using the Center Manifold Theorem and bifurcation theory (Elabbasy et al., 2014). Ghaziani, Govaerts, and Sonck have defined the conditions required for the stability of 3 balance points in the discrete system of functional Holling type (Ghaziani et al., 2012). In this type of analysis, discrete predator-prey systems have been observed to exhibit a much wider range of dynamic behaviors as compared to continuous predator-prey systems. Self-repeating processes are a matter of question in dynamic systems. The system holds periodical solutions in such cases. One of the most important theories among those that deal with the existence of periodical solutions is the theory developed by E.Hopf. (He et al, 2011). Tan \& Bora aimed for examining the dynamic behaviors of discrete predator-prey systems and modeling the predator-prey system that would reflect the actual system Korkmaz Tan and Bora, 2019). An algorithm for automatic parameter setting has been developed using the meta-intuitive algorithms, which were proved to be successful in many studies for solving the problems of chaotic systems. A predator-prey model has thus been created in which the species in an ecological system reflect the actual system behaviors of the existing species.

The emulation of dynamic systems is important for education and research purposes. Such an emulator should be designed in a cheap, robust, and quick way. FPGAs are used to emulate the neuron models given in (Gomar and Ahmadi, 2014; Chen et al., 2017). Unfortunately, not all the institutes or departments have FPGAs. Microcontrollers which are slower but cheaper than FPGAs are successfully used to emulate chaotic systems, ECKG signal generator and neurons in (Yener et al, 2017; Yener et al., 2017; Yener and Mutlu, 2018; Yener, 2019). A microcontroller-based memristive chaotic circuit is implemented in (Yener et al., 2017). A microcontroller-cased chaotic circuit of Lorenz Equations is designed in (Yener et al, 2017). An inexpensive microcontroller-based ECG signal generator is designed in (Yener and Mutlu, 2018). A microcontroller-based circuit is used to simulate two coupled Hindmarsh-Rose (HR) neurons in (Yener et al, 2017; Yener et al., 2017; Yener and Mutlu, 2018; Cagri, Yener). Lotka-Volterra equations can also be emulated with such circuits given in (Yener et al, 2017; Yener et al., 2017; Yener and Mutlu, 2018; Yener and Mutlu, 2019). To the best of our 
knowledge, this has not been done before. In this paper, a prey-predator emulator based on LotkaVolterra equations is designed using a STM32F429 Discovery Board. The circuit is made using cheap, rugged, easy-to-use components such as STM32F429 Discovery Board, two DA0832 digital analog converters (DACs), four LM 358 op-amps, and several passive components. The microcontroller solves the Lotka-Volterra differential equation system numerically, then sends two of the solved statevariables out as binary numbers throughout the digital ports to the DA0832 DACs, and obtains their analog waveforms using the opamp-based inverting amplifiers. Experimentally obtained waveforms of the emulator are also given in this study.

The STMStudio Program is used to scale the prey and predator state variables by adjusting the gains or the program constants. Then, the experimental circuit is assembled using cheap off-the shelves components on a breadboard and its waveforms in the time domain are obtained experimentally using a digital oscilloscope.

The paper is arranged as follows. In the second section, the Lotka-Volterra equations are briefly explained. In the third section, the microcontroller circuit board-based circuit of the Prey-predator Emulator circuit is introduced, the circuit is simulated with STMStudio, and its time-domain waveforms and phase portraits are given. In the fourth section, its experimental results are given. The paper is concluded with the last section.

\section{MATERIALS AND METHODS}

\section{Lotka-Volterra Equations}

The Lotka-Volterra equations are introduced in this section. The equations are given in both the continuous and the discrete time.

\section{Lotka-volterra equations in continuous time}

The following set of equations describes the Lotka-Volterra equations/system in the continuous time (Lotka, 1926):

$$
\begin{aligned}
& \frac{d x}{d t}=a x-b x y \\
& \frac{d y}{d t}=c x y-d y
\end{aligned}
$$

where $\mathrm{x}$ and $\mathrm{y}$ are the state variables presenting the numbers of prey (for example, rabbits) and predator (for example, wolves) populations, and tis time. $a, b, c$, and $d$ are Lotka-Volterra system parameters which are positive real numbers describing interaction of the two species.

\section{Lotka-volterra equations in discrete time}

In the discrete time, the Lotka-Volterra equations can be given as

$$
\begin{aligned}
& \frac{x[k-1]-[k]}{T_{s}} \cong a x[k]-b x[k] y[k] \\
& \frac{y[k+1]-y[k]}{T_{s}} \cong c x[k] y[k]-d y[k] \\
& x[k+1] \cong\left(1+a T_{s}\right) x[k]-b T_{s} x[k] y[k] \\
& y[k+1] \cong c T_{s} x[k] y[k]+\left(1-d T_{s}\right) y[k]
\end{aligned}
$$

where $T_{s}$ is the sampling time.

The discrete time system is used in the STM32F429 Discovery Board program to emulate the system. Euler's method is used to solve the equations in the discrete time. 


\section{Microcontroller-Based Emulator Circuit}

In this section, the circuit schematic of the microcontroller-based Lotka-Volterra System shown in Figure 1 is given and briefly explained. The circuit is made of a STM32F429 Discovery Board, two DA0832 digital analog converters (DACs), and two op-amp circuits used as the amplifiers of the DAC's. Feedback resistors of the OP1 and OP3 of the DAC ICs are chosen with proper resistance values to prevent saturation. The additional op-amps, OP2 and OP4, are used as op-amp based inverting amplifiers to have non-inverted state variables at the outputs. State variables of the LotkaVolterra equations, $x$ and $y$ are solved numerically using Euler's method. The two state variables are sent throughout the digital outputs to the DACs by STM32F429 Discovery Board as shown in Figure 3. The circuit is simulated in STMStudio. At the output of the inverting amplifiers fed by the DACs, the simulated state-variables obtained in the time-domain are shown in Figures 2 and 3.

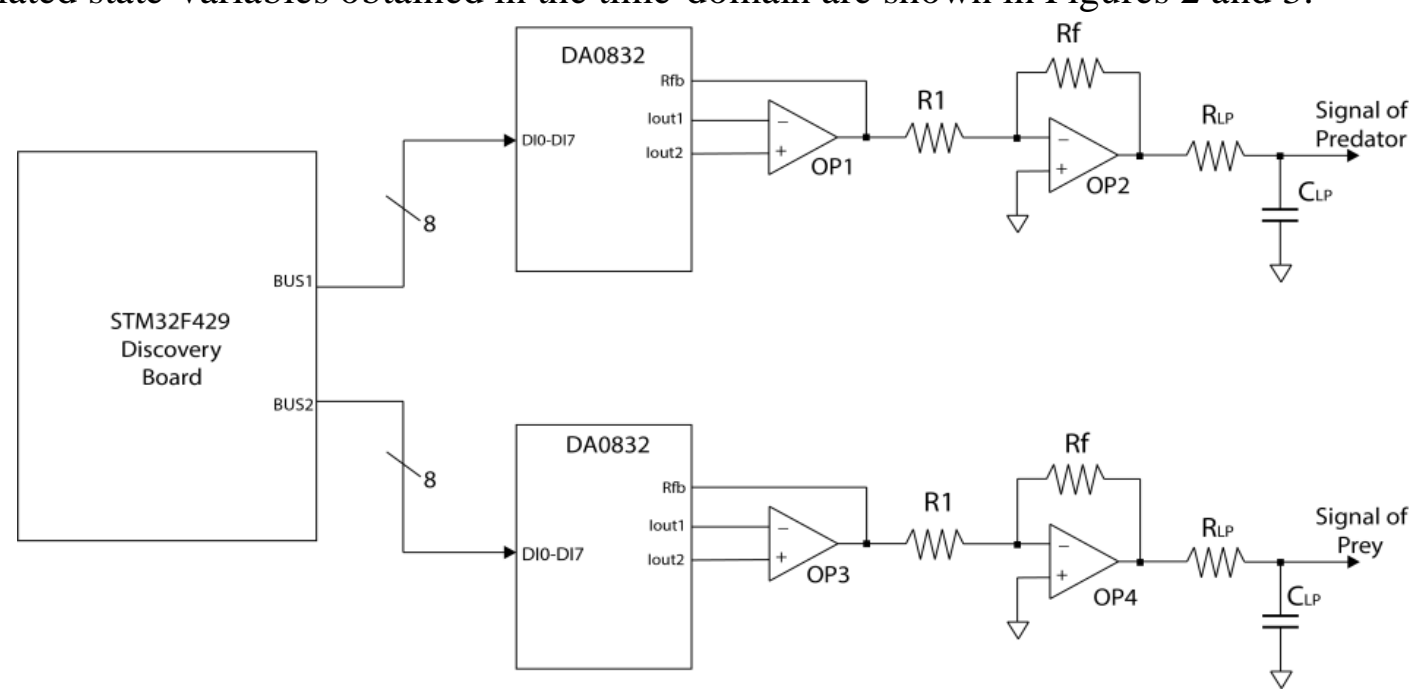

Figure 1. Circuit schematic of the microcontroller-based Lotka-Volterra system drawn.

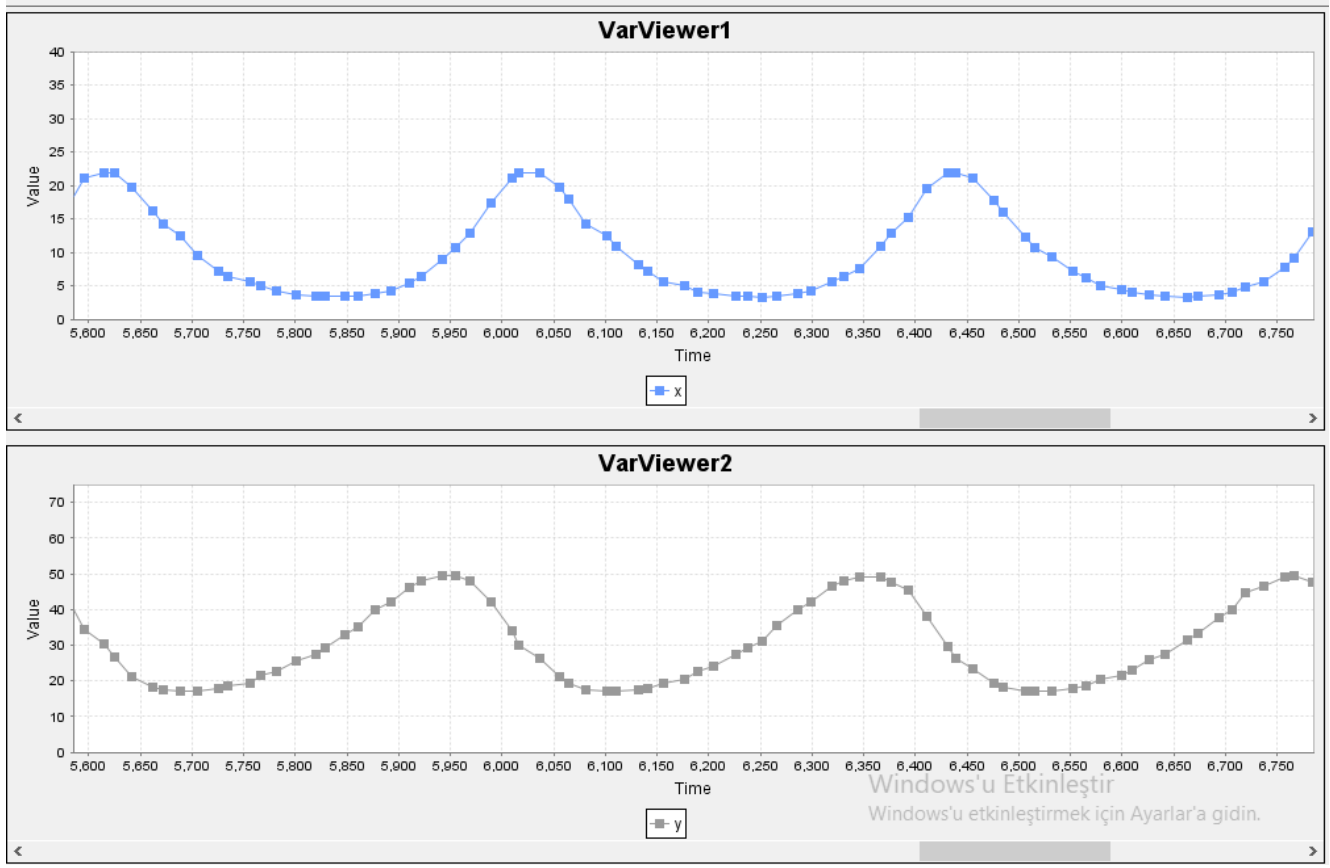

Figure 2. a) $x(t)$ and b) $y(t)$ state variables with respect to time simulated with $S T M S t u d i o$ for prd=10, pry=17, $a=0.3$, $\mathrm{b}=0.01, \mathrm{c}=0.1$, and $\mathrm{d}=0.01$. 

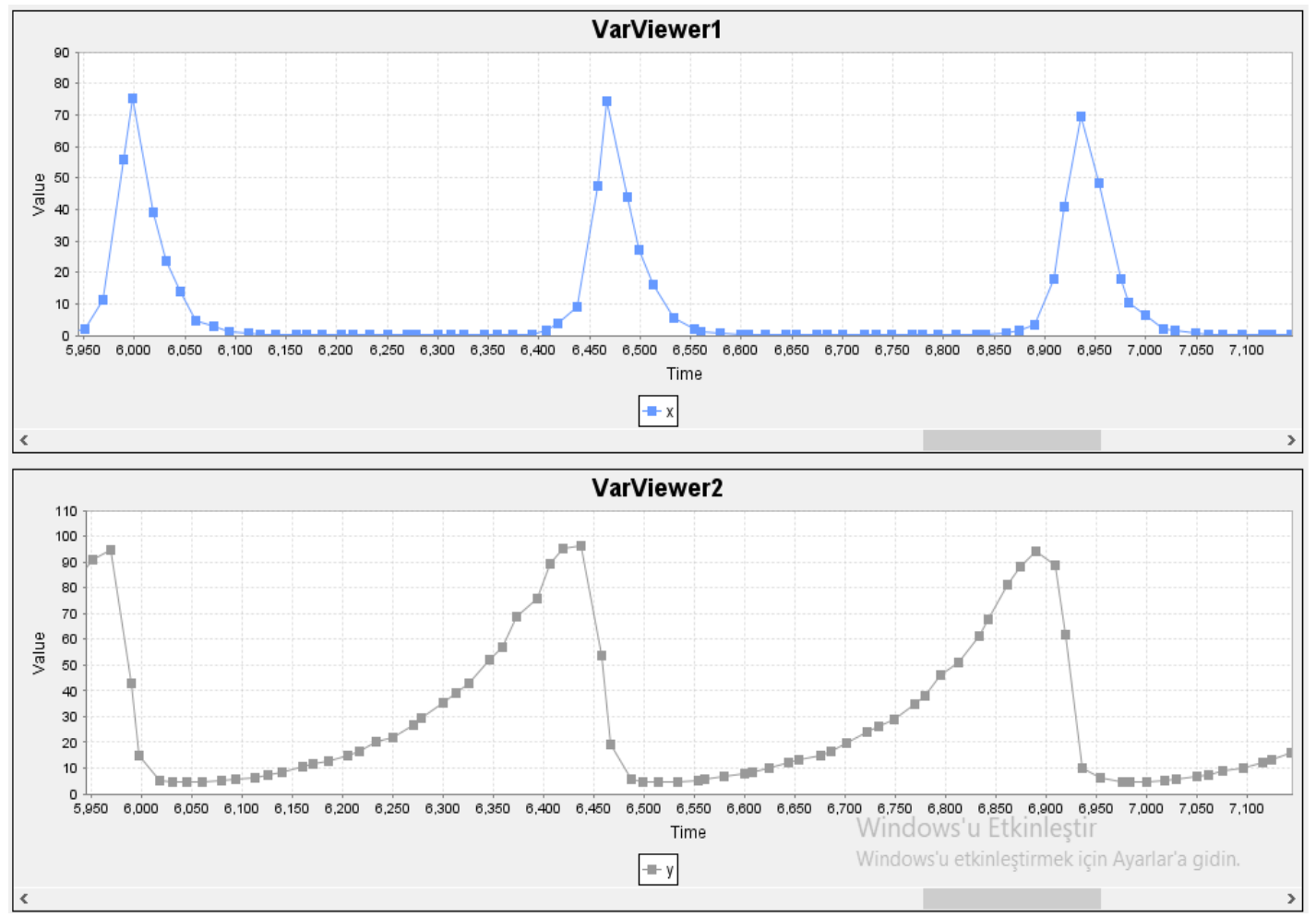

Figure 3. a) $x(t)$ and b) $y(t)$ state variables with respect to time simulated with STMStudio for $\operatorname{prd}=50$, pry $=50, a=0.5$, $b=0.02$, $c=0.1$, and $d=0.01$.

\section{RESULTS AND DISCUSSION}

Experimental results of the STM32F429 Discovery Board-based Lotka-Volterra circuit are given in this section. The circuit shown in Figure 4 is assembled. Mbed online compiler is used to compile the codes. The Lotka-Volterra system parameters are made adjustable using a TFT touchpad interface in the emulator circuit for entering parameters as inputs as shown in Figure 5. The prey and predator population signals are obtained with the designed emulator. All two state variables of Lotka-Volterra equations are sent to the output throughout the DACs and op-amps to obtain analog signals. Experimental output signals have been acquired by a $60 \mathrm{MHz}$ digital oscilloscope simultaneously at the DAC outputs and given in Figure 6 for different system parameters and initial values. The STM32F429 Discovery Board-based Lotka-Volterra emulator system is able to demonstrate prey and predator population behavior and its experimental waveforms, shown in Figures 6, match well to the simulation results shown in Figures 2 and 3. The prey waveform leads the predator waveform as shown in Figure 6. The shape of the population waveform varies with the initial conditions. Also, in Figure 6, it can be seen that a noise does exist within the waveforms due to the effect of quantization. The experimental phase-portraits or the limit cycles of the $x-y$ state variables of the circuit are shown in Figure 7 for different system parameters and initial values. It can be seen that the area of the limit cycle increases while the initial conditions of the prey and predator populations increases. The shape of the limit cycle varies with the initial conditions. It turns from a Baklava-like shape to a smoothened triangular-like shape as the initial conditions increases. Also, in Figure 7, it can be seen that a noise does exist within the limit cycle due to the quantization error. 


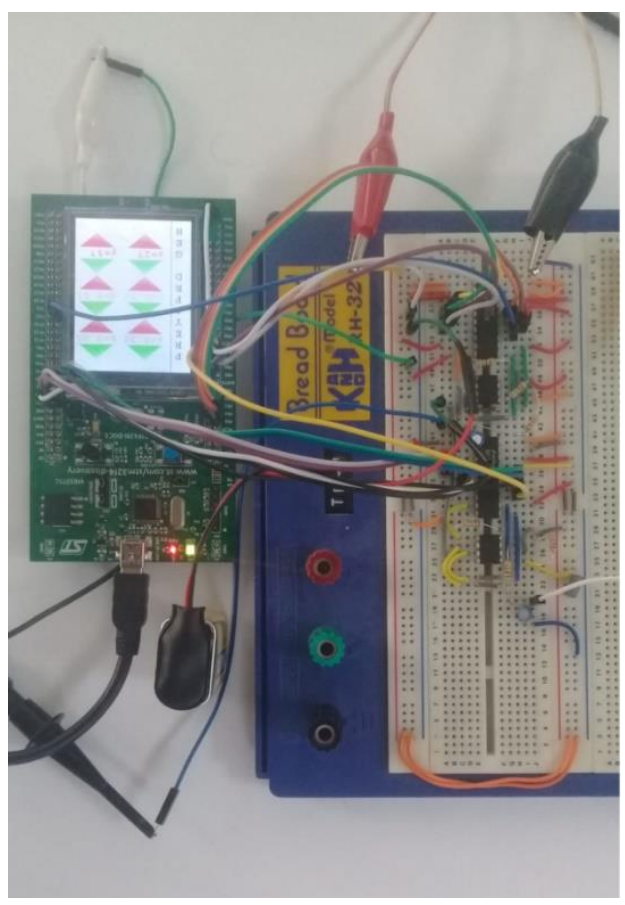

Figure 4. Photograph of the implemented STM32F429based Lotka-Volterra emulator circuit.

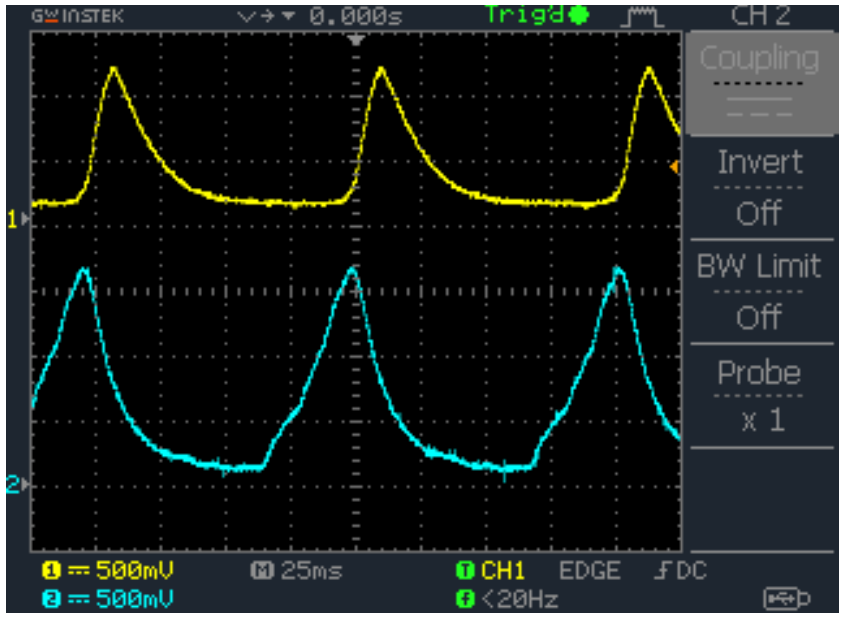

(a)

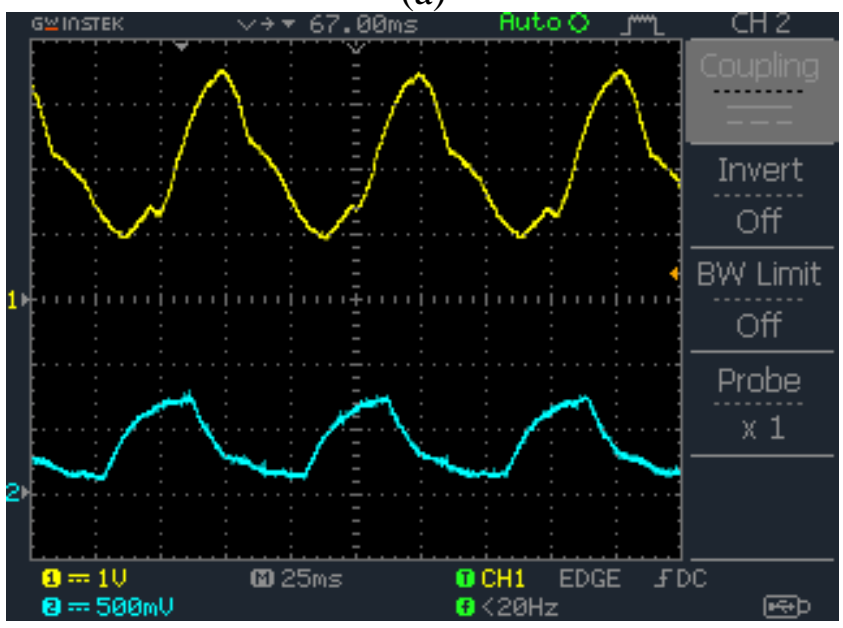

(c)

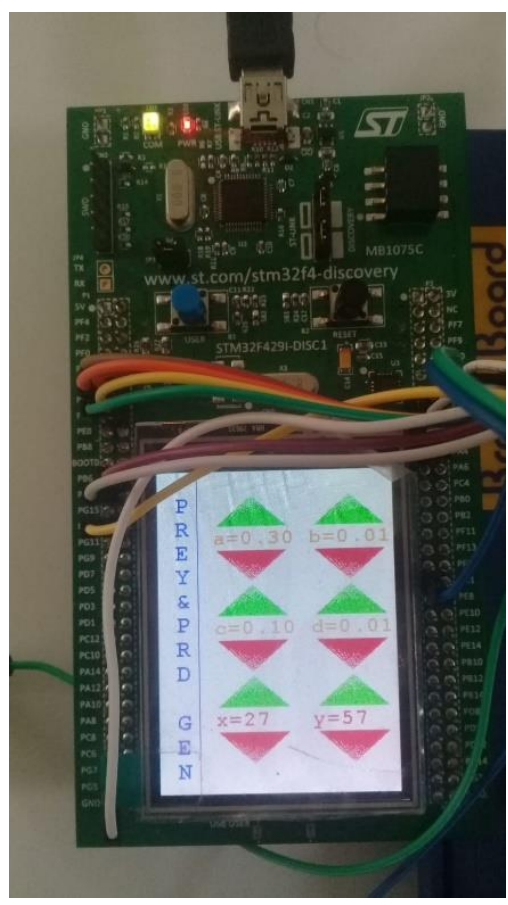

Figure 5. The TFT touchpad of the emulator used for parameter inputs.

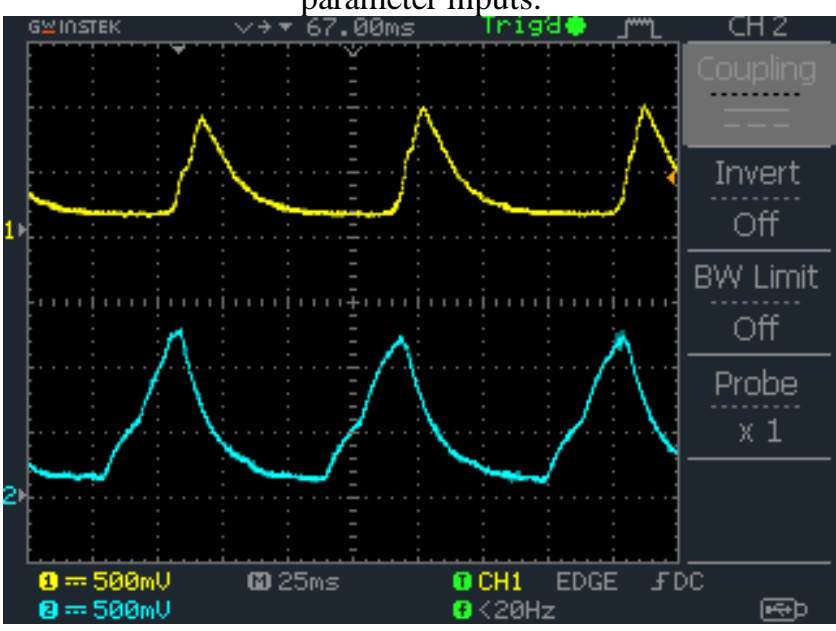

(b)

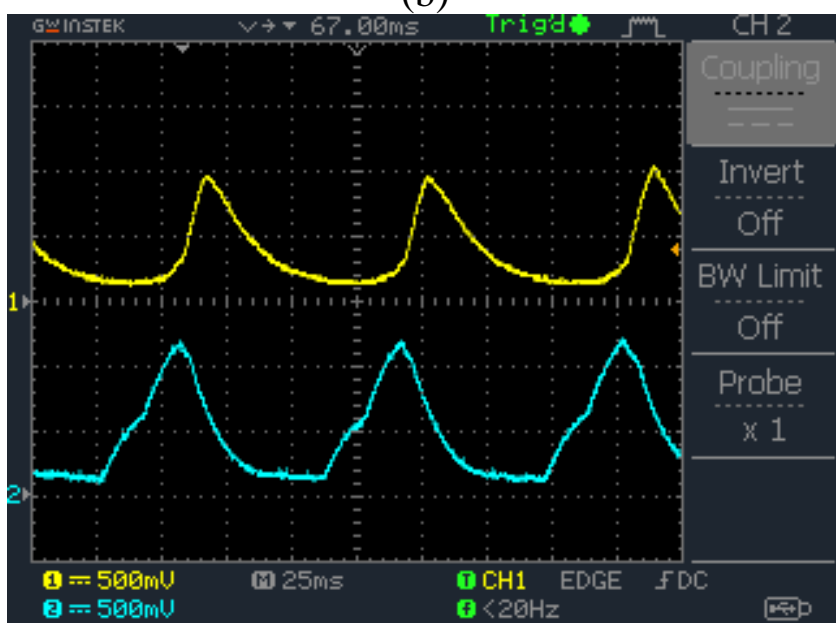

(d)

Figure 6. Prey-Predator waveforms: $x$ (yellow) and y (blue) versus time for a) prd=50, pry=50, $a=0.3, b=0.01, c=0.1$, and $\mathrm{d}=0.01, \mathbf{b})$ prd $=50, \mathrm{pry}=50, \mathrm{a}=0.5, \mathrm{~b}=0.02, \mathrm{c}=0.1$, and $\mathrm{d}=0.01, \mathbf{c})$ prd=50, pry=50, $\mathrm{a}=0.3, \mathrm{~b}=0.01, \mathrm{c}=0.1$, and $\mathrm{d}=0.001$, and d) $\operatorname{prd}=27$, pry $=57, \mathrm{a}=0.3, \mathrm{~b}=0.01, \mathrm{c}=0.1$, and $\mathrm{d}=0.01$ (pry and prd are the initial values of the prey and predator populations) 


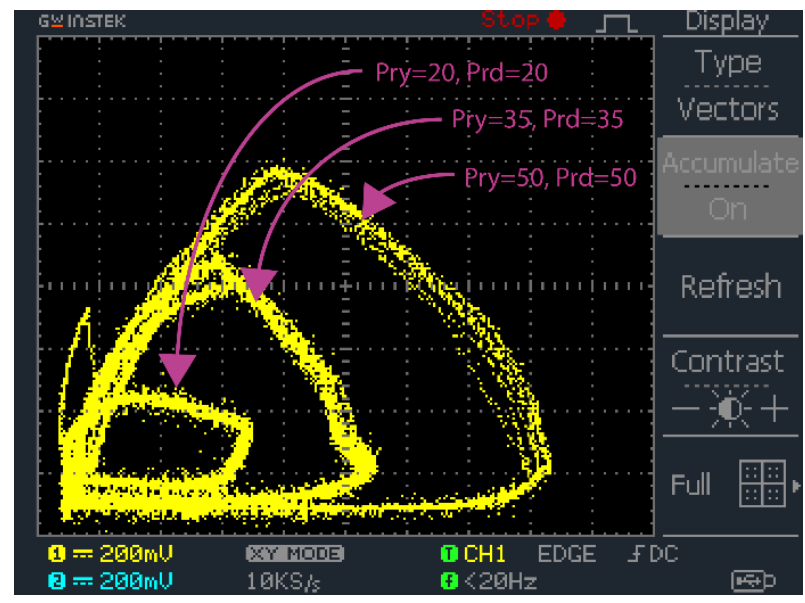

Figure 7. The phase-portraits or the limit cycles of the $x-y$ state variables of the circuit for $a=0.3, b=0.01, c=0.1$, $\mathrm{d}=0.01 \mathrm{prd}=20-35-50$, and $\operatorname{pry}=20-35-50$.

\section{CONCLUSION}

In this study, a STM32F429-based circuit of Lotka-Volterra emulator system is designed. The circuit is able to produce the scaled prey and predator signals obtained from the numerical simulations of Lotka-Volterra equations at the outputs of its inverting amplifiers. By using potentiometers connected to analog inputs of the STM32F429 Discovery Board, Lotka-Volterra system parameters are made to be adjustable and, therefore, the behavior of the system can be investigated easily for studies such as extinction and over-population. Such a circuit which is made of the cheap and powerful STM32F429 Discovery Board and off-the shelves components can be used for education and studies such as extinction, over-population, etc. More powerful emulators can be done using more powerful microcontrollers. Also, the limit of the species can be simulated using STM32F429 Discovery Board is an open research topic for the researchers.

\section{Conflict of Interest}

The article authors declare that there is no conflict of interest between them.

\section{Author's Contributions}

The authors declare that they have contributed equally to the article.

\section{REFERENCES}

Boshan, C, Jiejie, C, 2012. Bifurcation and chaotic behavior of a discrete singular biological economic system. Applied Mathematics and Computation 219, 5: 2371-2386.

Chen Q, Wang J, Yang S, Qin Y., Deng B., and Wei X, 2017. A real-time FPGA implementation of a biologically inspired central pattern generator network, Neurocomputing, 244, 63-80.

Elabbasy E M, Elsadany A A, and Zhang Y, 2014. Bifurcation analysis and chaos in a discrete reduced lorenz system. Applied Mathematics and Computation 228(2014), 184-194.

Ghaziani R K, Goverts K, Sonck C, 2012. Resonance and bifurcation a discrete time predator-prey system with Holling functional response. Nonlinear Analysis Real World Applications 13 (2012), 1451-1465.

Gleria IM, Figueiredo A, Rocha Filho, T M, 2001. Stability Properties of a General Class of Nonlinear Dynamical Systems. J.Phys A: Math. Gen. 34 3561-3575.

Goel NS, Maitra SC, and Montroll EW, 1971. On the Voltera and other nonlinear models of interacting populations. Rev. Modern Phys. 43, 231-276.

Gomar S, and Ahmadi A, 2014. Digital Multiplierless Implementation of Biological Adaptive-Exponential Neuron Model, IEEE Trans. Circuits Syst. I Regul. Pap., 61(4), 1206-1219. 
He X, Liao M. \& Xu C, 2011. Stability and Hopf Bifurcation analysis for a Lotka- Volterra predator-prey models with two delays, Int. J. Appl. Math. Comput., 21(1), 97- 107.

Kermack W O, and Mckendrick A G, 1927. A contribution to the mathematical theory of epidemics, Proceeding of Royal Society of London, Series A, Containing Papers of a Mathematical and Physical Character, 115(772), 700-72.

Kerner E H, 1957. The bulletin of mathematical biophysics. 19 (1957), 121;

Kimura M and Weiss G H, 1964. The stepping stone model of population structure and the decrease of genetic correlation with distance, Genetics 49 (1964), 561-576.

Korkmaz Tan R, and Bora Ş, 2019. Adaptive parameter tuning for agent-based modeling and simulation. Simulation: Transactions of the Society for Modeling and Simulation International, 95(9) 771-796.

Lotka A J, 1926. Elements of physical biology. Science Progress in the Twentieth Century, 21(82): 341-343.

Scheiner ER, 1996. Invitation to Dynamical Systems, The John Hopkins University Press, Washington, D.C.

Volterra V, 1978. Variazioni et fluttuazioni del numero d'individui in specie animali conviventi, Scudo ve Ziegler (Trans.), R. Comitato Talassografico Memoria, 6 (2), 31-113p.

Yener SC, Barbaros C, Mutlu R and Karakulak E, 2017. Implementation of Microcontroller-Based Memristive Chaotic Circuit, Acta Phys. Pol. A, 132(3), 1058-1061.

Yener ŞÇ, Barbaros C, Mutlu R and Karakulak E, 2018. Design of a Microcontroller-Based Chaotic Circuit of Lorenz Equations, in International Conference on Science and Technology ICONST 2018 5-9 September 2018 Prizren - KOSOVO, pp. 612-615.

Yener ŞÇ, Mutlu R, 2018. A Microcontroller-Based ECG Signal Generator Design Utilizing Microcontroller PWM Output and Experimental ECG Data, The Scientific Meeting on Electrical-Electronics Biomedical Engineering and Computer Science in 2018 (EBBT'2018) (18.04.2018-19.04.2018).

Yener SC, Mutlu R, 2019. - A Microcontroller Implementation Of Hindmarsh- Rose Neuron Model-Based Biological Central Pattern Generator, 1st International Informatics and Software Engineering Conference (UBMYK), 6-7 November 2019, Ankara.

Zhang G, Yi S and Boshan C, 2014. Bifurcation analysis in a discrete differential-algebraic predator-prey system. International Journal of Bifucation and Chaos, 38 (2014), 4559-4048. 